\title{
HARVESTING STRATEGIES FOR POPULATION SYSTEMS
}

\author{
FRED BRAUER
}

\begin{abstract}
We consider the effect of various types of harvesting on populations whose size is governed by a first order ordinary differential equation. We examine two problems - the possibility of catastrophe and the maximization of the harvest.
\end{abstract}

1. In studying the population size of some species of organisms, we may be led to consider the effect of harvesting (removal from the system) according to some rule. For example, we may wish to reduce the total population size of a nuisance species. Another possibility is that the species being harvested is useful, and we may be interested in controlling the size of the harvest. In such situations there are two principal concerns. One is the possibility of wiping out the species entirely, either by overharvesting to the point where the equilibrium population size is zero, or by setting up an unstable equilibrium with oscillations which may drive the population size to zero. This might be desirable if the species being harvested is a nuisance, but is certainly to be avoided if we are harvesting something of value and wish to continue to do so over a long time period. A class of examples of practical situations where we wish to avoid extermination of the species involves the setting of quotas for hunting or fishing.

The other aspect of harvesting which we shall examine is the question of maximizing the yield without endangering the species. We shall not consider this problem from an economic point of view involving costs and returns, as in [5], but shall seek only the maximum safe harvest.

By restricting ourselves to populations governed by a single first order differential equation, we rule out some possibilities which may arise for more complicated models. For example, predator-prey systems exhibit some behaviour which is impossible for one-dimensional systems. Other questions which can be answered analytically for one-dimensional systems can only be studied numerically for predator-prey systems. Some of these questions are currently being studied jointly with A. C. Soudack.

2. We consider the ordinary differential equation

$$
y^{\prime}=F(y),
$$

Received by the editors on December 1, 1977.

AMS (MOS) subject classification (1970). 92A15, 34D05.

Copyright (C) 1979 Rocky Mountain Mathematics Consortium 
where $F(y)$ is differentiable for $y \geqq 0$, as a model for the size $y(t)$ of a population. This assumes that the growth rate $F(y(t))$ of the population size at time $t$ depends only on $y(t)$, even though this neglects many factors which may be significant for real populations. Some of these factors were suggested in [2]. It is natural to assume

$$
F(0)=0,
$$

that is, that the growth rate of the population size vanishes when the population size is zero. This implies that if the population size is zero at any time, then it remains zero for all future time. In fact, by the uniqueness theorem for differential equations, a solution of (1) is either identically zero or is always different from zero.

We assume further that there exists a "carrying capacity" $K$ such that

$$
F(K)=0,
$$

$F(y)>0[0<y<K], F(y)<0[y>K]$. This implies $F^{\prime}(K)<0$, and therefore that the equilibrium solution $y=K$ of (1) is asymptotically stable. Also, $F^{\prime}(0)>0$, and therefore the equilibrium solution $y=0$ of (1) is unstable. It follows easily that every solution of (1) with positive initial value tends to the limit $K$ as $t \rightarrow \infty$; hence the name "carrying capacity" for $K$.

The model (1) describes the population size if there is no harvesting. We now consider the effect of removing members from the population at a time rate $H(y, E)$ which depends on the population size and on a non-negative parameter $E$ which may be regarded as a harvesting effort. We shall assume that zero effort produces no harvest for any population size

$$
H(y, 0)=0, \quad H_{y}(y, 0)=0,
$$

that for a given effort the harvest increases with population size but its rate of increase decreases,

$$
H(y, E) \geqq 0, H_{y}(y, E) \geqq 0, \quad H_{y y}(y, E) \leqq 0,
$$

and that for a given population size the harvest increases with effort but its rate of increase decreases,

$$
H_{E}(y, E) \geqq 0, \quad H_{E E}(y, E) \leqq 0 .
$$

Here, subscripts denote partial derivatives. For future reference, we note that since $H_{y}$ is a decreasing function of $y$,

$$
H(y, E)=\int_{0}^{y} H_{y}(z, E) d z \geqq y H_{y}(y, E) .
$$


We assume further that $H(y, E)$ is bounded uniformly in $y$ on $0 \leqq y \leqq K$ for each $E \geqq 0$, but that $H(y, E) \rightarrow \infty$ as $E \rightarrow \infty$ for each $y>0$. However, we should point out that the two cases which have been studied most extensively, $H(y, E)=E[1,6]$ and $H(y, E)=E y[3$, $4,5]$, do not satisfy all of these hypotheses. On the other hand, the forms most frequently hypothesized for predator response, such as $H(y, E)=E y /(y+A)[7]$ or $H(y, E)=E y\left(1-e^{-c y}\right)[8,9]$ do satisfy the above hypotheses, and harvesting may be regarded as human predation.

The population size under harvesting is described by the differential equation

$$
y^{\prime}=F(y)-H(y, E) .
$$

The equilibrium solutions of (7) are the solutions $y_{\infty}(E)$ of

$$
F\left(y_{\infty}\right)=H\left(y_{\infty}, E\right)
$$

and an equilibrium $y_{\infty}(E)$ is asymptotically stable if

$$
F^{\prime}\left[y_{\infty}(E)\right]<H_{y}\left[y_{\infty}(E), E\right] .
$$

Geometrically, this says that an equilibrium is given by an intersection of the curves $u=F(y)$ and $u=H(y, E)$, and an equilibrium $y_{\infty}$ is asymptotically stable if the crossing is such that the second curve goes from below the first curve to above the first curve as $y$ increases. Since $H(y, E) \geqq 0$ and $F(y) \geqq 0$ only for $0 \leqq y \leqq K$, any equilibrium $y_{\infty}$ satisfies $0 \leqq y_{\infty} \leqq K$. The assumption $H(0, E)=0$ implies that $y=0$ is an equilibrium which is asymptotically stable if $F^{\prime}(0)<H_{v}(0, E)$ and unstable if $F^{\prime}(0)>H_{y}(0, E)$. Since $H_{y}(y, 0)=0$ the equilibrium $y=0$ is unstable for small $E>0$. Under the assumptions (2), (3), (4), (5), every solution of (7) tends to an equilibrium as $t \rightarrow \infty$. However, if $H(y, 0)>0$, as for constant-rate harvesting, $H(y, E)=E$, there may be no equilibrium, if $H(y, E)>F(y)$ for all $y \geqq 0$, in which case every solution of (7) decreases monotonically to zero in finite time. This raises the possibility of a mathematical catastrophe-a situation where the limit as $t \rightarrow \infty$ of a solution of (7) is a discontinuous function of $E$. As has been shown in [1], this always occurs for constant rate harvesting. It may also happen that as $E$ increases, $y_{\infty}(E)$ tends continuously to zero. Since this corresponds to an eventual collapse of the population, it may be described as a biological catastrophe. More precisely, we say that a mathematical catastrophe occurs if there is a critical effort $E_{c}$ such that

$$
\lim _{E \uparrow E_{c}} y_{\infty}(E)>0, y_{\infty}(E)=0 \quad\left[E>E_{c}\right],
$$


and a biological catastrophe occurs if there is a critical effort $E_{c}$ such that

$$
\lim _{E \uparrow E_{c}} y_{\infty}(E)=0
$$

In either case $(10)$ or $(11)$,

$$
F^{\prime}\left[y_{\infty}\left(E_{c}\right)\right]=H_{y}\left[y_{\infty}\left(E_{c}\right), E_{c}\right]
$$

as well as

$$
F\left[y_{\infty}\left(E_{c}\right)\right]=H\left[y_{\infty}\left(E_{c}\right), E_{c}\right] ;
$$

the assumption that $\lim _{E \rightarrow \infty} H(y, E)=\infty$ for $y>0$ implies that for sufficiently large $E$ there is no non-zero equilibrium, and thus there must be a catastrophe of some kind. In a practical sense, a mathematical catastrophe is more dangerous than a biological catastrophe since it gives no warning of its approach as $E$ increases; it would be useful to have some criteria to predict which type of catastrophe will occur for a given model, or function $F$ and a given harvesting scheme, or function $H$.

A population model is said to exhibit depensation [5] if there is an interval $0<y<a$ on which

$$
F(y)<y F^{\prime}(y) .
$$

Theorem 1. A population with depensation with a harvesting scheme which satisfies the above hypotheses (3), (4), and (5) always exhibits a mathematical catastrophe.

Proof. Let $E$ be any effort which produces an asymptotically stable equilibrium $y_{\infty}(E)$ in the interval $0<y<a$ in which (14) is satisfied. Then, because of (9), (14), and (8)

$$
\begin{aligned}
H_{u}\left[y_{\infty}(E), E\right] & \geqq F^{\prime}\left[y_{\infty}(E)\right] \\
& >\frac{F\left[y_{\infty}(E)\right]}{y_{\infty}(E)} \\
& =\frac{H\left[y_{\infty}(E)\right]}{y_{\infty}(E)},
\end{aligned}
$$

but this contradicts (6). Thus $y_{\infty}(E)$ cannot be in the interval $0<y<a$, and there must be a discontinuity in $y_{\infty}(E)$.

If there is no depensation, a population may or may not exhibit a mathematical catastrophe, depending on the harvesting scheme. For constant-rate harvesting, $H(y, E)=E$ (which fails to satisfy the hypoth- 
esis (3)) there is always a mathematical catastrophe [1]. For logistic growth, $F(y)=r y(1-(y / K))$, and the harvesting scheme $H(y, E)=$ $E y^{1 / 2}$ it is easy to calculate that there is a mathematical catastrophe for $E_{c}=\left(2 / 3^{1 / 3}\right) r K^{1 / 2}$, corresponding to $\mathrm{y}_{\infty}\left(E_{c}\right)=K / 3$. On the other hand, for logistic growth and proportional harvesting, $H(y, E)=E y$ there is no mathematical catastrophe, as $y_{\infty}(E)$ tends continuously to zero [5].

3. We wish now to consider the question of how to maximize the harvest in the following sense. Let $y_{\infty}(E)$ be a non-zero asymptotically stable equilibrium of (7), so that solutions $y(t)$ tend to $y_{\infty}(E)$ as $t \rightarrow \infty$. Since the rate at which members of the population are harvested at time $t$ is $H[y(t), E]$, this harvest rate tends as $t \rightarrow \infty$ to $H\left[y_{\infty}(E), E\right]$. We define the yield

$$
Y=H\left[y_{\infty}(E), E\right],
$$

the harvest rate when the system is in equilibrium. Since every solution of (7) tends to an equilibrium as $t \rightarrow \infty$, this yield is the long-term average harvest rate, $\lim _{T \rightarrow \infty}(1 / T) \int_{0}^{T} H[y(E), E] d t$. Our goal is to maximize $y$ as a function of $E$.

TheоReм 2. Let $M$ be the maximum value of $F(y)$ on $0 \leqq y \leqq K$, attained for $y=y_{M}$. If $E$ can be taken large enough to make $y_{\infty}(E)=y_{M}$, then the value $E_{M}$ for which $y_{\infty}\left(E_{M}\right)=y_{M}$ maximizes $y$, the maximum value of $Y$ is $M$, and the equilibrium at $y_{M}$ is asymptotically stable.

Proof. Because of (8), when the system (7) has an equilibrium at $y_{\infty}$, the yield $Y$ is $F\left(y_{\infty}\right)$. Thus $M$ is the maximum possible yield, attained if $E$ can be chosen to put the equilibrium at $y_{M}$. Since $F^{\prime}\left(y_{M}\right)=0$ and by assumption $H_{u}\left(y_{M}, E\right)>0$, it follows immediately from (9) that an equilibrium at $y_{M}$ is asymptotically stable.

Whether the maximum yield is attainable depends on the natures of $F$ and $H$. If $H(y, E)=E$, the maximum yield is attainable, but only when the system is on the verge of catastrophe [1]. If $H(y, E)=E y$, then $y_{\infty}(E)$ decreases continuously from $K$ to 0 as $E$ increases, and the maximum yield is attained without danger of catastrophe. The essential content of Theorem 2 is that the maximum yield obtainable is the maximum rate of growth of population size, independent of the method of harvesting, and that this yield, since it corresponds to an asymptotically stable equilibrium, can be achieved in practice.

Theorem 2 gives a theoretical answer to the question of how to maximize the yield. In practice, other methods of harvesting might be used which could conceivably produce a larger yield. For example, a prohibition of harvesting when the population is low could allow the population to build up rapidly, so that most of the time the population is 
large and the harvest correspondingly large. Another possibility, an extreme case of this, would be to allow harvesting only during a short "hunting season" during which a specified number of members of the population are removed-what might be called a bang-bang harvesting process. The population is then allowed to build up for the next hunting season. Such a process is best modelled by a differential equation of the form (1) with no harvesting term, the harvesting being taken care of by changing the initial conditions and re-starting the system. We will show in the next section that the yield cannot be increased by any such method.

Another possibility would be a harvest rate which depends explicitly on time, $H(t, y, E)$. For such a scheme we must define the yield as the long-term average harvest rate,

$$
Y=\lim _{T \rightarrow \infty} \frac{1}{T} \int_{0}^{T} H[t, y(t), E] d t,
$$

where $y(t)$ is the solution of the differential equation

$$
y^{\prime}=F(y)-H(t, y, E) .
$$

This is required, because solutions of (15) do not necessarily tend to an equilibrium as $t \rightarrow \infty$. For example, if $H$ is periodic in $t$ there may be periodic solutions.

If $y(t)$ is a solution of (15), integration of (15) from 0 to $T$ gives

$$
\int_{0}^{T} H[t, y(t), E] d t=\int_{0}^{T} F(y(t)) d t-y(T)+y(0) .
$$

If $\mathrm{y}(\mathrm{t})$ is bounded for $0 \leqq t<\infty$,

$$
\lim _{T \rightarrow \infty} \frac{y(T)}{T}=\lim _{T \rightarrow \infty} \frac{y(0)}{T}=0,
$$

and thus

$$
\begin{aligned}
Y & =\lim _{T \rightarrow \infty} \frac{1}{T} \int_{0}^{T} H[t, y(t), E] d t \\
& =\lim _{T \rightarrow \infty} \frac{1}{T} \int_{0}^{T} F[y(E)] d t \\
& \leqq \max _{0 \leqq y \leqq K} F(y) .
\end{aligned}
$$

Therefore a time-dependent harvest can not produce a larger yield than an autonomous one. 
4. If $y(t)$ is a solution of $(1)$, then the time $T$ required for $y(t)$ to increase from $y_{0}-H$ to $y_{0}$ is given by

$$
\int_{v_{0}-M}^{y_{0}} \frac{d u}{F(u)}
$$

obtained by separation of variables. Thus if $H$ members are removed from the population when the population size is $y_{0}$ and the population is then allowed to grow back to size $y_{0}$, the time rate of harvesting is

$$
\frac{H}{T}=H / \quad \int_{y_{0}-H}^{y_{0}} \frac{d u}{F(u)} .
$$

By the mean value theorem,

$$
\int_{y_{0}-H}^{y_{0}} \frac{d u}{F(u)}=\frac{H}{F(\eta)}
$$

for some $\eta, y_{0}-H<y_{0}$, and thus the harvesting rate is $F(\eta) \leqq \max _{0 \leqq y \leqq K} F(y)$. This shows that a bang-bang harvest can not produce a greater yield than the maximum for a continuous harvest as given by Theorem 2. More generally, this argument shows that a closed season method of harvesting can not increase the yield because the recovery time is not short enough to make up for the closed season.

This reasoning may also be used to set hunting quotas. If we specify a time interval 1 between hunting seasons, and a population $y_{0}$ at the beginning of the hunt, then the permissible harvest $H$ is given by

$$
\int_{y_{0}-H}^{y_{0}} \frac{d u}{F(u)}=1
$$

For example, if the population growth is logistic, $f(y)=r y(1-y / K)$, evaluation of the integral in (16) leads to the formula

$$
H=\frac{y_{0}\left(K-y_{0}\right)\left(e^{r}-1\right)}{y_{0}+\left(K-y_{0}\right) e^{r}} .
$$

If we apply this to the sandhill crane model studied in [1], with $K=$ $194,600, r=0.0987$, we obtain the following relation between $y_{0}$ and $H$.

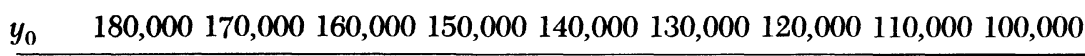

\begin{tabular}{llllllllll}
\hline$H$ & 1,390 & 2,200 & 2,900 & 3,480 & 3,960 & 4,330 & 4,590 & 4,750 & 4,800 \\
\hline
\end{tabular}

TABLE 1 
This table may be compared with the results of [1] giving equilibrium populations for various harvest rates.

5. A more refined description of a population system may be given by a predator-prey system. We think of the predator as the species in which we are interested and the prey as the food supply on which it depends. Such a system is modelled by a pair of first ordinary differential equations, which admits a richer variety of qualitative possibilities than a single first order equation. For example, there may be periodic orbits, which give oscillatory behaviour for the (predator) population size. There are significant differences between the one and two-dimensional cases. For a predator-prey system, the maximum yield may correspond to an unstable equilibrium, in which case this theoretical yield has no practical meaning. The proofs that time-dependent and bangbang harvests can not produce a greater yield are inherently onedimensional and cannot be adapted to predator-prey systems. The only apparent way to determine whether a bang-bang harvest can produce a larger yield is by a numerical simulation. The question remains open, but is currently being explored in some joint work with A. C. Soudack.

\section{REFERENCES}

1. F. Brauer and D. A. Sánchez, Constant rate population harvesting: equilibriuum and stability, Theoretical Population Biology 8 (1975), 12-30.

2. F. Brauer, A. C. Soudack, and H. S. Jarosch, Stabilization and de-stabilization of predator-prey systems under harvesting and nutrient enrichment, Int. J. Control 23 (1976), $553-573$.

3. F. Brauer and A. C. Soudack, Response of predator-prey systems to nutrient enrichment and proportional harvesting, Int. J. Control 27 (1978), 65-86.

4. C. W. Clark, Mathematical Bioeconomics, Conference on some Mathematical Problems in Biology, P. von den Driessche, ed., Springer-Verlag Lecture Notes in Biomathematics 2 (1974), 29-45.

5. — Mathematical Bioeconomics, Wiley, New York (1976).

6. B. S. Goh, Optimal control of a fish resource, Malayan Scientist 5 (1969-70), 65-70.

7. C. S. Holling, The functional response of predators to prey density and its role in mimicry and population regulation, Mem. Entom. Soc. Canada 45 (1965), 1-60.

8. V. S. Ivlev, Experimental ecology of the feeding of fishes, Yale University Press, New Haven, 1961.

9. M. L. Rosenzweig, Paradox of enrichment: destabilization of exploitation ecosystems in ecological time, Science 171 (1971), 385-387.

Department of Mathematics, University of Wisconsin, Madison, Wi 53706 